\title{
Effet du son de blé et de la nature des lipides du régime sur la digestibilité, l'activité des enzymes digestives et la lipémie des porcelets méditerranéens de la race Alentejana
}

\author{
JPB Freire ${ }^{1}$, J Peiniau 2, LF Cunha 1, \\ JAA Almeida ${ }^{3}$, A Aumaitre ${ }^{2}$ \\ 1 Instituto Superior de Agronomia, Tapada da Ajuda, 1399 Lisboa codex, Portugual \\ 2 Station de recherches porcines, Centre de recherche Inra de Saint-Gilles, \\ 35550 L'Hermitage, France \\ 3 Universidade de Évora, Nucleo da Mitra, 7000 Evora, Portugal
}

(Reçu le 28 juin 1995 ; accepté le 4 décembre 1995)

\begin{abstract}
Résumé - Au total, 24 porcelets de la race Alentejana (six animaux par traitement) sevrés à 21 jours ont été utilisé pour l'étude de l'adaptation digestive et métabolique des animaux à l'utilisation du son de blé (0 ou $15 \%$ ) et à la nature des lipides (huile d'olive ou suif incorporés à $5 \%$ ) du régime de sevrage. Les régimes expérimentaux, isoénergétiques et isoazotés, sont formulés pour deux niveaux de polysaccharides non amylacés (NDF $=10$ ou $16 \%$ ) et deux sources de lipides selon un schéma factoriel $2 \times 2$. Des prélèvements sanguins sont réalisés par cathétérisation jugulaire (à jeûn et au cours des 5 heures qui suivent le repas) à 45 jours d'âge. À la fin de l'expérience les porcelets sont abattus pour le contrôle du dévelopement du tube digestif et le prélèvement du pancréas et de la muqueuse intestinale. L'addition de son de blé entraîne une baisse significative $(p<0,01)$ de la digestibilité (CUD) de l'énergie de 3 et de 6 points de pourcentage avec l'huile d'olive et le suif, respectivement. Les CUD de la matière sèche et de l'énergie sont augmentés de 2 et de 4,5 points en réponse à l'addition de l'huile d'olive. Le CUD des lipides est en moyenne significativement plus élevé $(\rho<0,01)$ pour les rations à base d'huile d'olive (70\%) que pour le suif (52\%). De plus la présence de son de blé entraîne une réduction du CUD des lipides de 65,4 à 56,9. L'inclusion du son de blé a augmenté de 18 et de 13 points le CUD du NDF et des hémicelluloses, seulement en présence d'huile d'olive, d'où l'existence d'une interaction significative $(p<0,01)$ entre la teneur en fibres et la nature des lipides sur la digestibilité de ces fractions. Avec le suif, la digestibilité des glucides pariétaux a été indépendante de la teneur en NDF du régime. Enfin l'addition de fibres ou de lipides modifie peu le poids des organes digestifs: seul le poids du pancréas augmente avec la teneur en fibres. L'activité totale et spécifique des enzymes digestives du pancréas n'est pas significativement modifiée par la composition du régime. La teneur en fibres et la nature des lipides n'ont pas modifié les paramètres biochimiques du sérum sanguin (glucose, urée, cholestérol, HDL-cholestérol, LDL-cholésterol et triglycérides). L'urémie observée à jeun est presque doublée $(p<0,01) 3$ heures après l'ingestion d'aliment. Enfin les porcelets Alentejano ont
\end{abstract}


montré une bonne capacité de régulation du cholestérol sanguin même lorsque leur régime supplémenté en suif est enrichi en cholestérol.

porc Alentejano / sevrage / glucides pariétaux / lipides / physiologie digestive / cholestérol

Summary - Effect of level of wheat bran and fat source on digestive performance and serum cholesterol of Alentejano weaned piglets. Twenty-four piglets of the Alentejano breed weaned at 3 weeks of age were randomly affected to four treatments including two levels of wheat bran ( 0 or $15 \%)$ and two sources of fat ( $5 \%$ of olive oil or $5 \%$ of beef tallow) in a $2 \times 2$ factorial arrangement. The effects of the level of wheat bran and fat source (olive oil or beef tallow) were evaluated on growth performance, faecal apparent digestibility (FAD) and activity of pancreatic and intestinal digestive enzymes. In addition, the effect of the diet on the serum level of glucose, urea, cholesterol and triglycerides was measured prior and during $5 \mathrm{~h}$ after an experimental meal. The inclusion of wheat bran to the diet decreased the FAD of energy by 3 and 6 percentage units in the presence of olive oil and tallow, respectively. A further reduction in the FAD of ether extract was observed when animals were fed wheat bran 65.4 versus $56.9 \%$, respectively. There is a significant $(P<0.01)$ interaction between fibre level and fat source on the FAD of NDF and hemicellulose, so the addition of wheat bran increased the $F A D$ of these fractions only in the presence of olive oil. Compared to the diet containing beef tallow, FAD of dry matter and energy were increased by 2 and 4.5 percentage units by the addition of olive oil in the diet: Moreover, the FAD of ether extract was significantly higher $(P<0.01)$ for the diets containing olive oil (70\%) than for those supplemented with tallow (52\%). Despite an increase in the weight of the pancreas in piglets fed diets supplemented with wheat bran, total or specific activity of pancreatic enzymes were not significantly affected by the composition of the diet. The level of total cholesterol, $H D L$ cholesterol and LDL cholesterol and that of triglycerides were not affected by the addition of tallow and consequently by the increase in dietary cholesterol.

Alentejano pigs / weaning / fibre / fat source / digestive physiology / serum cholesterol

\section{INTRODUCTION}

Les animaux de race Alentejana sont des porcs de type méditerranéen élevés au sud du Portugal dans un système agro-sylvopastoral qui comporte une alimentation riche en fibres et de nombreuses périodes de sous-alimentation. Jusqu'aux années 1950 ces animaux de génotype gras ont constitué une des principales sources alimentaires (viande et graisses) pour les populations du sud du Portugal. Cependant une adiposité excessive de la carcasse et l'apparition en 1957 de la peste porcine africaine au Portugal ont fait reculer les effectifs. Ainsi au début des années 1980 on ne dénombrait plus que 5000 truies Alentejanas (Antunes Correia et Oliveira, 1985). Néanmoins, à partir de 1986, on assiste à un intérêt renouvelé pour l'élevage d'animaux de cette race, en raison de la qualité de leurs produits (jambons, produits de saucisserie) et de leur capacité à valoriser les ressources naturelles des «Montados». Les porcs de race Alentejana sont connus pour bien valoriser un régime composé d'herbe et de glands, donc riche en fibres et en lipides insaturés. Mais on ne connaît pas dans quelle mesure la variation de la teneur en fibre et de la composition des lipides du régime traditionnel peut améliorer l'efficacité nutritionnelle des animaux. En effet chez le porc de race améliorée on sait que l'augmentation de la teneur en glucides pariétaux du régime réduit la croissance (Campbell et Taverner, 1986), la digestibilité de l'énergie et de l'azote du régime (Henry, 1976). Par ailleurs, l'utilisation de lipides augmente la concentration énergétique du régime et peut améliorer les performances zootechniques des animaux (Jones et al, 
1992). De plus les graisses les plus saturées comme le suif sont moins hydrolysables in vitro (Aumaitre et Rérat, 1968) et moins digérées par le porcelet (Eusébio et al, 1965 ; Braude et Newport, 1973) comparativement aux graisses insaturées apportées par les huiles.

La connaissance de l'effet de la teneur en glucides pariétaux et de la nature des graisses du régime sur la physiologie digestive et le métabolisme glucidique et lipidique des porcs Alentejano constitue un problème majeur. Chez les animaux de génotype maigre, l'ingestion de glucides pariétaux peut stimuler la sécrétion pancréatique (Corring et al, 1989) parallèlement à un effet hypoglycémiant (Bengala Freire et al, 1990). Par ailleurs, de nombreux travaux ont démontré chez l'homme que l'addition de fibres dans la ration avait un effet hypocholestérolémiant en diminuant l'absorption intestinale du cholestérol (Jenkins et al, 1985). Enfin, l'activité de la lipase pancréatique augmente avec la teneur en lipides du régime et la lipémie change avec la nature et la qualité des graisses ingérées (Sethi et al, 1993).

Dans ce travail nous avons utilisé des porcelets de race Alentejana pour étudier les conséquences digestives et métaboliques de l'utilisation de deux niveaux de glucides pariétaux (10 et $16 \%$ de NDF) et deux sources de lipides (huile d'olive, suif) dans le régime distribué dès le sevrage.

\section{MATÉRIEL ET MÉTHODES}

À 3 semaines d'âge, quatre lots de six porcelets (trois mâles et trois femelles) de la race Alentejana ont été constitués selon un schéma factoriel $2 \times 2$. La répartition des animaux par bloc dans les traitements tient compte du poids, du sexe et de l'origine de la portée. Les animaux ont reçu un des quatre régimes dont la composition est rapportée au tableau I. Dans les régimes, équilibrés en énergie brute et en azote, on a utilisé le son de blé à raison de 0 ou $15 \%$ et
I'huile d'olive ou le suif comme source de lipides à un taux unique de $5 \%$. Les régimes, formulés pour satisfaire les besoins en protéines, énergie digestible et acides aminés indispensables (Inra, 1984), ont été distribués en alimentation égalisée pour les animaux d'un même bloc entre 3 et 7 semaines d'âge sous la forme de granulés ( $3 \mathrm{~mm}$ de diamètre). Après 3 jours d'adaptation au régime, les porcelets ont été soumis à deux périodes consécutives de 7 jours de mesures et de collectes individuelles d'excreta. Les fèces et les urines ont été collectées pour analyse et calcul de la digestibilité fécale apparente et du bilan azoté. Les échantillons collectés ont été mélangés et lyophilisés avant broyage avec un broyeur à marteaux muni d'une grille de $1 \mathrm{~mm}$ (Retsch 5657 Haan, Allemagne). À 42 jours d'âge, les porcelets ont été opérés (cathétérisation jugulaire) permettant ainsi un prélèvement sanguin après un jeune de 12 heures et six prélèvements (30 minutes, 60 minutes, 90 minutes, 3 heures, 4 heures et 5 heures après la distibution de l'aliment) consécutifs à un repas de 1 heure. À chaque prise $5 \mathrm{~mL}$ de sang ont été prelevé. Trois jours après les collectes de sang, les animaux ont été abattus pour la mesure du développement de l'appareil digestif et le prélèvement du pancréas. La muqueuse de l'intestin grêle a été prélevée et un échantillon moyen a été préparé et immédiatement stocké au froid $\left(-25^{\circ} \mathrm{C}\right)$ avant l'analyse.

La matière sèche a été dosée par dessication à $105^{\circ} \mathrm{C}$ et les minéraux à $550^{\circ} \mathrm{C}$, l'azote par la méthode de Kjeldhal. L'énergie a été mesurée à la bombe calorimétrique adiabatique Parr 1261 (Parr, Moline, II, États-Unis), les lipides par extraction à l'éther après hydrolyse acide $(\mathrm{HCl} 3 \mathrm{~N})$ et les glucides pariétaux par la méthode de $V$ an Soest avec désamidonage préalable (Van Soest, 1963) grâce à un appareil automatic Fibertec (Högönas, Suède). La lipase, la trypsine et la chymotrypsine pancréatiques ont été dosées selon les méthodes de Rathelot et al (1975), Bergmeyer et Gawehn (1978), et Reboud et al (1962), respectivement. L'amylase a été dosée par spectrophotométrie selon la méthode de Métais et Bieth (1968) et les disaccharidases intestinales par colorimétrie d'après Dahlquist (1964). Les paramètres sanguins ont été dosés sur le plasma par des méthodes enzymatiques: le glucose selon la méthode de la glucose-oxydase (Biomérieux, ref 61272), l'urée par la réaction de Berthelot à l'uréase (Boeheringer, ref 777510), les triglycérides par colorimétrie (Biomérieux, ref 61236), le cholestérol par la cholestérol estérase, 
Tableau I. Composition centésimale et chimique des régimes.

Lipides

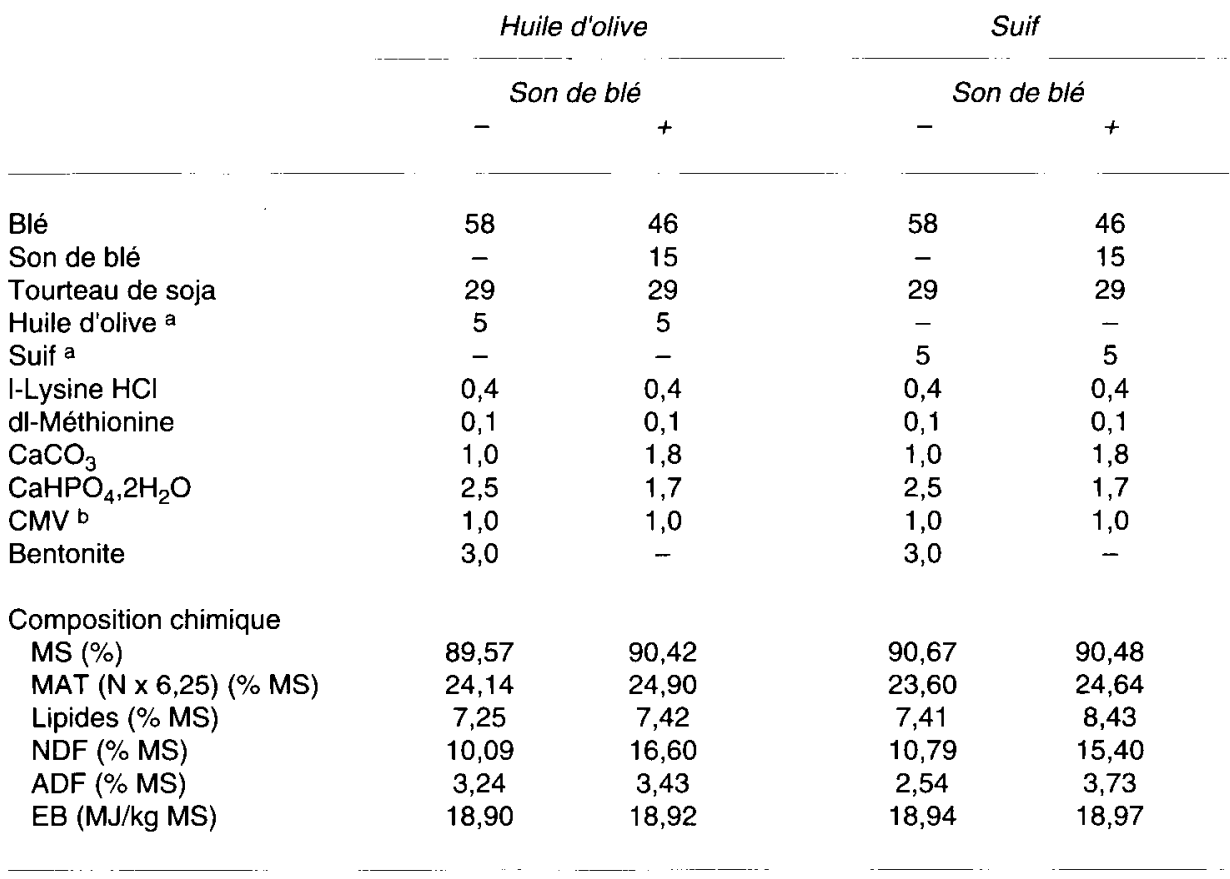

a Huile d'olive : $0,0014 \mathrm{~g}$ cholestérol $/ \mathrm{kg}$, suif : $1 \mathrm{~g}$ cholestérol $/ \mathrm{kg}$ (Inra, 1987). b Le composé minéral vitaminique apporte par kg d'aliment : Vit A : $5400 \mathrm{UI}$; vit D3 : $1080 \mathrm{UI}$; vit E : 7,5 mg; vit K3 : 0,725 mg; vit B1 : 0,2 mg ; vit B2 : $1,125 \mathrm{mg}$; vit $B 6: 0,0650 \mathrm{mg}$; Biotine : $0,01 \mathrm{mg}$; Ac folique : 0,175 ; Ac nicotinique : 7,75 mg ; Ac pantothénique : $7,75 \mathrm{mg} ; \mathrm{Fe}: 47,5 ; \mathrm{Cu}: 10 \mathrm{mg} ; \mathrm{Mn}: 21,5 \mathrm{mg} ; \mathrm{Zn}: 50,0 \mathrm{mg} ; \mathrm{Mg}: 50,0 \mathrm{mg} ; \mathrm{I}: 0,195 \mathrm{mg} ; \mathrm{Co}: 10 \mathrm{mg} ; \mathrm{Se}: 0,055$ $\mathrm{mg}$; Choline : $50 \mathrm{mg}$; Excipient : $1 \mathrm{~g}$

la cholestérol oxydase et la peroxydase (Biomérieux, ref 61224), le LDL-cholestérol par précipitation des LDL suivie par la solubilisation et le dosage enzymatique du cholestérol et les HDLcholestérol par élimination des LDL et le dosage du cholestérol associé aux lipoprotéines de haute densité par voie enzymatique (Biomérieux, ref 61351).

Les résultats de croissance, digestibilité fécale apparente (CUD) et bilan azoté ont été analysés en prenant l'effet de la teneur en fibres $(F)$, la source de lipides (L) et l'âge de l'animal ou période (P) comme facteurs de variation. L'activité des enzymes digestives et le poids des organes à l'abattage ont été comparés selon un schéma fac- toriel $(2 \times 2)$. On évalue ainsi l'effet de la teneur en fibres $(F)$, de la source de lipides $(L)$ et de l'interaction $(F \times L)$. L'effet de la teneur en fibres et de la source de lipides du régime sur les paramètres sanguins a été comparé par analyse de variance selon un modèle en split-plot. La pratique de plusieurs prises de sang dans le temps sur chaque porcelet fait qu'on considère l'erreur associée aux blocs comme résiduelle pour tester l'effet des régimes. Les analyses de variance ont été faites à l'aide du logiciel Sas (1989) utilisant la procédure GLM. Enfin les données des paramètres sanguins sont essentiellement représentés graphiquement, en faisant figurer les coefficients de variation (CV) et le résultat de l'analyse de variance. 
Tableau II. Effet de la teneur en fibres, de la nature des lipides du régime et de la période sur les performances zootechniques des procelets Alentejanos.

\begin{tabular}{|c|c|c|c|c|c|c|c|c|c|c|c|c|}
\hline \multirow{3}{*}{. } & \multicolumn{2}{|c|}{ Huile d'olive } & \multicolumn{2}{|c|}{ Suif } & \multicolumn{2}{|c|}{ Période $\mathrm{b}$} & \multicolumn{6}{|c|}{ Analyse de variance a } \\
\hline & \multicolumn{2}{|c|}{ Son de blé } & \multicolumn{2}{|c|}{ Son de blé } & \multirow[t]{2}{*}{1} & \multirow[t]{2}{*}{2} & \multirow[t]{2}{*}{$F$} & \multirow[t]{2}{*}{$L$} & \multirow[t]{2}{*}{$P$} & \multirow[t]{2}{*}{$F \times L$} & \multirow[t]{2}{*}{ ETR } & \multirow[t]{2}{*}{$C V R$} \\
\hline & - & + & - & + & & & & & & & & \\
\hline Poids initial (kg) & 4,3 & 4,2 & 4,1 & 4,3 & - & - & & & & & 0,44 & 0,10 \\
\hline Poids final $(\mathrm{kg})$ & 5,9 & 6,1 & 6,0 & 5,8 & - & - & & & & & 0,60 & 0,10 \\
\hline Ingéré (g/jour) & 368 & 425 & 412 & 406 & 355 & 446 & & & $\star \star \star$ & & 47 & 0,12 \\
\hline Gain moyen quotidien (g) & 122 & 135 & 132 & 127 & 116 & 142 & & & ** & & 30,8 & 0,24 \\
\hline Indice de consommation & 3,2 & 3,3 & 3,2 & 3,4 & 3,2 & 3,3 & & & & & 0,64 & 0,20 \\
\hline
\end{tabular}

a Effets : $F$ : teneur en fibres, $L$ : nature des lipides, $P$ : période, NS : non significatif, ${ }^{* *} p<0,01$. Pour toutes les variables interactions : $F \times L: N S, F \times P: N S, L \times P: N S$. ETR : Écart type de la résiduelle. CVR : coefficient de variation de la résiduelle. ${ }^{b}$ Période $1: 25$ à 32 jours, période $2: 32$ à 38 jours d'âge.

\section{RESULTATS}

Les résultats des performances zootechniques (tableau II) montrent que, pour une même quantité d'aliment ingéré, la nature des lipides et le niveau des glucides pariétaux du régime exprimé par la teneur en NDF et ADF n'ont modifié ni la croissance journalière ni l'indice de consommation des animaux.

L'incorporation de $15 \%$ de son de blé dans le régime a fait baisser le coefficient d'utilisation digestive (CUD) de l'énergie (E) de 3 et de 6 points avec l'huile d'olive et le suif respectivement $(p<0,01)$ (tableau III). Cependant l'effet négatif du son de blé sur l'énergie digestible (ED) du régime atteint le seuil de la signification statistique seulement avec le suif : baisse de $3 \%$ avec l'huile d'olive $(p>0,05)$ et de $7 \%$ avec le suif $(p<0,05)$. L'addition d'huile d'olive comme source de lipides a augmenté $(p<0,01)$ de deux points la digestibilité de la matière sèche (MS), de 4,5 points la digestibilité de l'énergie et de $0,85 \mathrm{MJ} / \mathrm{kg}$ MS la teneur en ED du régime. L'effet de la nature des lipides sur la digestibilité fécale apparente de l'azote est fonction du taux de glucides pariétaux du régime (interaction fibres $x$ lipides significative $p<0,05$ ). L'utilisation d'huile d'olive a augmenté la digestibilité de l'azote avec les régimes les plus riches en fibres. Le CUD des lipides est en moyenne significativement plus élevé pour les rations à base d'huile d'olive $(70 \%)$ que pour celles à base de suif $(52 \%)(p<0,05)$, la présence de son de blé entraînant une réduction du CUD des lipides de 65,4 à 56,9\%. La digestibilité des glucides pariétaux est très variable (CVR $=0,15$ pour le NDF et 0,96 pour l'ADF). Avec l'huile d'olive, la digestibilité des fractions NDF et hémicelluloses augmente avec l'incorporation de son de blé, ce qui ne se produit pas avec le suif (tableau III). En revanche avec le suif, l'augmentation de la teneur en NDF du régime est sans effet sur la digestibilité du NDF et des hémicelluloses. En deuxième période les digestibilités de la matière sèche, de l'énergie et de l'azote sont significativement améliorées. Enfin les résultats du bilan azoté montrent que le coefficient de rétention azotée (CRN) et le coefficient d'utilisation pratique de l'azote (CUPN) sont indépendants du taux de glucides pariétaux et 
Tableau III. Effet de la teneur en fibres, de la nature des lipides du régime et de la période sur la digestibilité totale apparente, l'énergie digestible et le bilan azoté.

\begin{tabular}{|c|c|c|c|c|c|c|c|c|c|c|c|c|}
\hline & Huile & d'olive & & uif & & iode & & & alys & $e d e v$ & ariance & \\
\hline & Son c & de blé & Son & de blé & 1 & 2 & $F$ & $L$ & $P$ & $F \times L$ & ETR & $C V R$ \\
\hline & - & + & - & + & & & & & & & & \\
\hline CUD (\%) & & & & & & & & & & & & \\
\hline Matière sèche & $78,7^{a}$ & $79,5^{a}$ & $78,1^{\mathrm{a}}$ & $75,7^{b}$ & 76,8 & 79,2 & & ** & ** & * & 2,1 & 0,03 \\
\hline Énergie & $81,6^{a}$ & $78,8^{b}$ & $78,5^{b}$ & $72,6^{c}$ & 75,8 & 79,9 & ** & ** & ** & & 3,0 & 0,04 \\
\hline Matière organique & $84,6^{a}$ & $82,9^{a}$ & $82,8^{a}$ & $79,1^{b}$ & 81,1 & 83,6 & ** & ** & $\star \star$ & & 1,9 & 0,02 \\
\hline Azote & $78,3^{a}$ & $80,9^{a}$ & $77,5^{\mathrm{ab}}$ & $74,5^{b}$ & 75,0 & 80,5 & & ** & ** & * & 4,0 & 0,05 \\
\hline Lipides & $72,9^{a}$ & $66,6^{\mathrm{a}}$ & $57,8^{b}$ & $47,2^{c}$ & 55,5 & 66,8 & ** & $\star \star \star$ & $\star \star$ & & 8,7 & 0,14 \\
\hline NDF & $43,8^{a}$ & $61,7^{b}$ & $52,6^{c}$ & $53,6^{c}$ & 54,3 & 51,6 & ** & & & ** & 7,7 & 0,15 \\
\hline ADF & 12,8 & 11,4 & 9,3 & 18,0 & 16,6 & 9,2 & & & & & 12,6 & 0,96 \\
\hline NDF-ADF & $60,8^{a}$ & $73,3^{b}$ & $66,9^{c}$ & $64,8^{a c}$ & 66,7 & 66,2 & * & & & ** & 6,6 & 0,10 \\
\hline $\begin{array}{l}\text { Énergie digestible } \\
\text { (MJ/kg MS) }\end{array}$ & $15,4^{a}$ & $15,0^{\mathrm{ab}}$ & $14,9^{b}$ & $13,8^{c}$ & 14,3 & 15,1 & ** & ** & ** & & 0,6 & 0,04 \\
\hline CRN (\%) & 55,4 & 62,4 & 58,7 & 61,5 & 61,0 & 58,0 & & & & & 8,1 & 0,14 \\
\hline CUPN $(\%)$ & 43,1 & 50,4 & 45,4 & 45,8 & 45,8 & 46,6 & & & & & 7,0 & 0,15 \\
\hline
\end{tabular}

1 Effets : $F$ : teneur en fibres, $L:$ nature des lipides, $P$ : période, NS : non significatif, ${ }^{\star} p<0,05,{ }^{* \star}: p<0,01$. Pour toutes les variables des interactions: F $\times P$ : NS, $L \times P$ : NS, F $x$ L $\times P$ : NS. ETR : Écart type de la résiduelle. CVR : coefficient de variation de la résiduelle. a,b Les valeurs affectées d'une même lettre sur une même ligne ne sont pas significativement différentes.

de la nature des lipides du régime (tableau III).

Le poids ( $\mathrm{g} / \mathrm{kg}$ poids vif) des organes de l'appareil digestif est rapporté au tableau IV. Le poids de l'intestin grêle plein est réduit de $18 \%$ consécutivement à l'association du son de blé avec l'huile d'olive. En revanche, le son de blé associé au suif a augmenté $(p<0,05)$ de $18 \%$ le poids du gros intestin plein. Dans les régimes à base d'huile d'olive, le son de blé a augmenté de $18 \%$ le poids du pancréas. L'activité totale exprimée par gramme de tissu frais et l'activité spécifique des enzymes digestives du pancréas et de la muqueuse intestinale des porcelets (tableau V) n'est pas significativement modifiée, sauf pour l'invertase, par la teneur en fibres ou la nature des lipides du régime.
La teneur en glucides pariétaux et la nature des lipides du régime n'ont pas significativement affecté $(p>0,05)$ les constituants lipidiques du plasma : le cholestérol, l'HDL-cholestérol, le LDL-cholestérol et les triglycérides (figs 1, 2 et 3). En revanche, le taux circulant de cholestérol augmente après le repas pour atteindre un maximum 90 minutes après l'ingestion d'aliment (fig 2). La variation du taux de triglycérides est fonction de la nature des lipides ingérés : avec l'huile d'olive on trouve un pic 60-90 minutes après le repas alors qu'avec le suif il n'y a pas de véritable augmentation du taux de triglycérides (fig 2). Le taux d'urée sanguin est doublé $(p<0,01) 3$ heures après la distribution d'aliment indépendamment de la nature du régime (fig 1). Enfin le taux basal du glucose est relativement élevé, seul le 
Tableau IV. Effet de la teneur en fibres et de la nature des lipides du régime sur le développement ( $\mathrm{g} / \mathrm{kg}$ de poids vif) de l'appareil digestif des porcelets.

\begin{tabular}{|c|c|c|c|c|c|c|c|c|c|c|}
\hline & \multirow{2}{*}{\multicolumn{2}{|c|}{$\begin{array}{l}\text { Huile d'olive } \\
\text { Son de blé }\end{array}$}} & \multirow{2}{*}{\multicolumn{2}{|c|}{$\begin{array}{c}\text { Suif } \\
\text { Son de blé }\end{array}$}} & \multicolumn{6}{|c|}{ Analyse de variance ${ }^{1}$} \\
\hline & & & & & \multirow[t]{2}{*}{$P t$} & \multirow[t]{2}{*}{$F$} & \multirow[t]{2}{*}{$L$} & \multirow[t]{2}{*}{$F \times L$} & \multirow[t]{2}{*}{ ETR } & \multirow[t]{2}{*}{ CVR } \\
\hline & - & + & - & + & & & & & & \\
\hline Poids à l'abattage $(\mathrm{kg})$ & 8,3 & 8,1 & 7,8 & 7,4 & ** & & & & 1,15 & 0,15 \\
\hline \multicolumn{11}{|l|}{ Estomac } \\
\hline Plein & 17,8 & 17,8 & 20,2 & 22,7 & & & & & 10,6 & 0,54 \\
\hline Vide & $9,8^{a}$ & $10,8^{\mathrm{ab}}$ & $11,0^{a b}$ & $11,9^{\mathrm{b}}$ & * & & * & & 1,25 & 0,11 \\
\hline \multicolumn{11}{|l|}{ Intestin grêle } \\
\hline Plein & $42,0^{a}$ & $34,4^{b}$ & $35,9 a b$ & $37,8^{\mathrm{ab}}$ & & & & * & 4,9 & 0,13 \\
\hline Vide & 31,6 & 29,7 & 31,1 & 33,4 & * & & & & 2,9 & 0,09 \\
\hline \multicolumn{11}{|l|}{ Gros intestin } \\
\hline Plein & $65,7^{a b}$ & $60,2^{a b}$ & $57,9^{\mathrm{a}}$ & $68,7^{b}$ & $\star \star \star$ & & & * & 7,3 & 0,12 \\
\hline Vide & 27,6 & 27,6 & 26,7 & 30,3 & & & & & 3,1 & 0,12 \\
\hline Foie & 32,6 & 31,6 & 34,1 & 33,3 & & & & & 3,9 & 0,12 \\
\hline Pancreas & $2,49^{a}$ & $2,94^{b}$ & $2,67^{a b}$ & $2,80^{\mathrm{ab}}$ & & * & & & 0,32 & 0,12 \\
\hline Rate & 1,85 & 1,87 & 2,36 & 1,91 & & & & & 0,38 & 0,19 \\
\hline
\end{tabular}

1 Effets : Pt : portée, F : teneur en fibres, $\mathrm{L}:$ nature des lipides, ${ }^{*}: p<0,05,{ }^{\star *}: p<0,01$. ETR : écart type de la résiduelle. CVR : coefficient de variation de la résiduelle. a,b Sur une même ligne les valeurs affectées d'une même lettre ne sont pas significativement différentes.

régime suif + son de blé entraîne une valeur inférieure à 100 mg/100 mL (fig 1).

\section{DISCUSSION}

\section{Digestibilité fécale apparente des constituants de la ration}

Nos résultats confirment l'effet classique des polysaccharides non amylacés (PNA) du régime sur la digestibilité de la matière sèche et de l'énergie (Henry 1976). Par ailleurs, l'utilisation des lipides mono-insaturés de l'huile d'olive a permis de compenser partiellement l'effet négatif des PNA sur le contenu en énergie digestible du régime : réduction de $3 \%(p>0,05)$ avec l'huile d'olive et de $7 \%(p<0,05)$ avec le suif (tableau III). L' interaction significative teneur en fibres $x$ nature des lipides sur le CUD de l'azote recoupe les résultats de Chabeauti et al (1991) et permet de soupçonner un effet différent de l'huile d'olive et du suif sur l'activité de la flore microbienne du gros intestin (Frank, 1982). Enfin, la baisse de l'utilisation digestive des lipides avec le son de blé est le résultat d'une excrétion endogène accrue (Drochner, 1984).

La baisse de digestibilité de l'énergie trouvée après addition de suif s'explique par la moindre utilisation digestive de la fraction lipidique (tableau III). Ces résultats sont classiques (Eusébio et al, 1965) et en 
Tableau V. Effet de la teneur en fibres et de la nature des lipides du régime sur l'activité totale (Ul/g tissu) et spécifique (UI/mg de protéine) des enzymes pancréatiques et intestinales des porcelets.

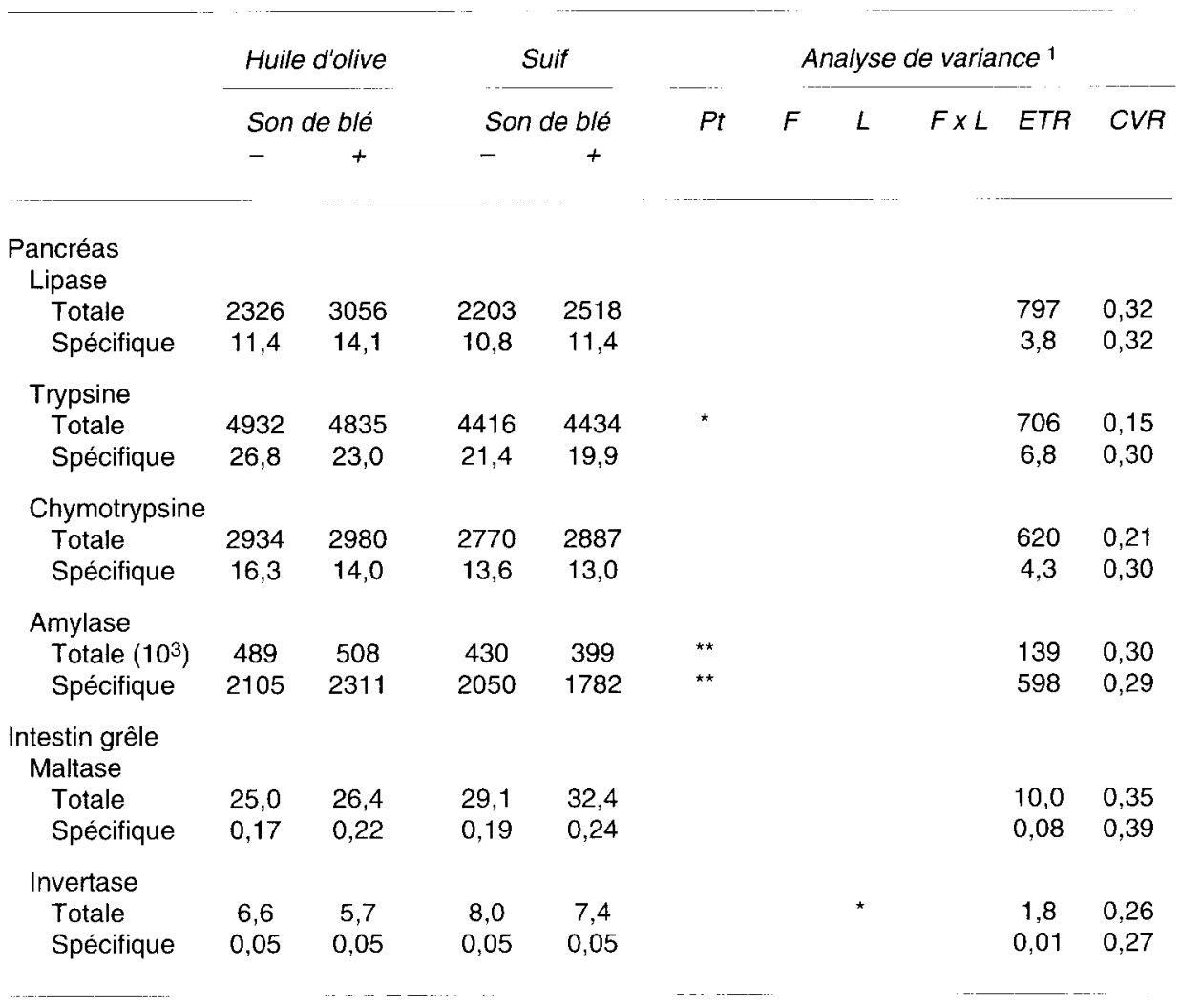

1 Effets : Pt : portée, $F$ : teneur en fibres, $L:$ nature des lipides, ${ }^{*}: p<0,05,{ }^{*}: p<0,01$. ETR : Écart type de la résiduelle. CVR : coefficient de variation de la résiduelle.

accord avec la moindre vitesse d'hydrolyse in vitro du suif par rapport au saindoux et à l'huile d'olive (Aumaitre et Rérat, 1968). Cependant il est bien connu chez le porc que les mesures de digestibilité fécale apparente des lipides ont une signification nutritionelle limitée (Enders et al, 1988) tout en affectant significativement la teneur en énergie digestible de la ration. Les valeurs de la digestibilité fécale apparente des fractions NDF, ADF et hémicelluloses sont comparables à celles rapportées par Peiniau (1984) et Bengala Freire et al (1990) sur des animaux de race Large White. Ceci paraît confirmer que les porcelets Alentejano ne sont pas plus efficaces dans l'utilisation digestive des parois végétales et recoupe les résultats de Février et al (1992). La digestibilité des parois végétales de la ration est enfin fonction de leur niveau d'incorporation et de leur nature (Peiniau, 1984, Bengala Freire et al, 1990). L'absence d'effet sur la digestibilité du NDF et des hémicelluloses du son de blé observé en présence de suif peut s'expliquer par un effet négatif de l'arrivée d'une plus grande quantité de lipides indigérés au gros intestin sur l'activité des bactéries dégradant ces 

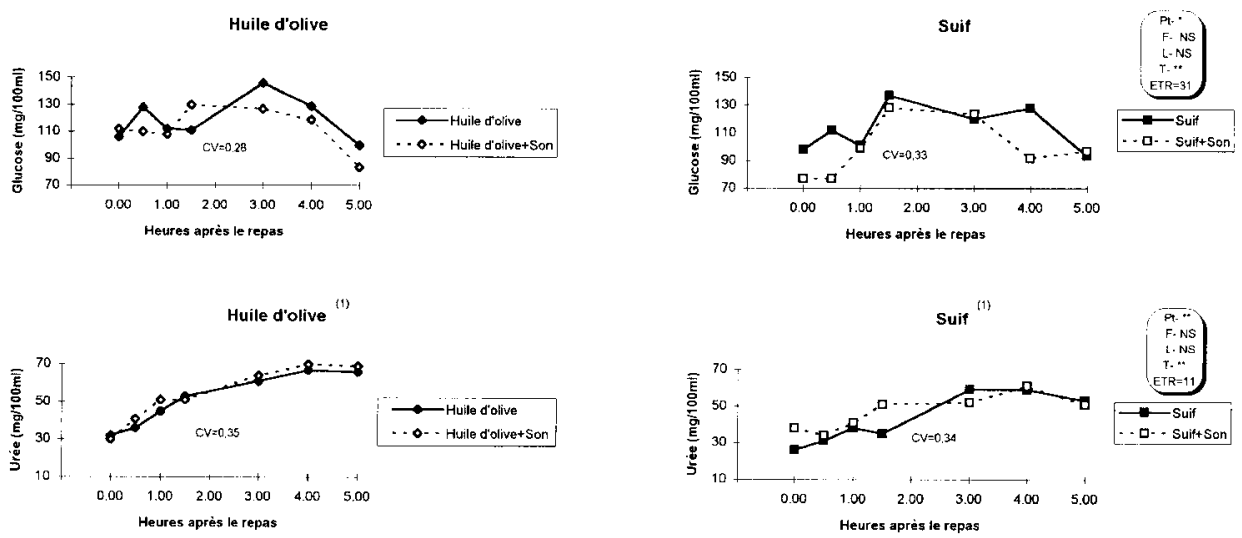

Fig 1. Effet de la nature des lipides et de la présence de fibres dans le régime sur la variation postprandiale de la glycémie et de l'urémie. Effets : Pt : portée, $\mathrm{F}:$ taux de fibres, $\mathrm{L}:$ nature des lipides, $\mathrm{T}$ : temps après le repas, ETR : écart type de la résiduelle, $\mathrm{CV}$ : coefficient de variation.

(1) L'addition de son entraîne une augmentation significative $(p<0,05)$ du taux postprandial d'urée.

composés. L'augmentation $(p>0,05) \mathrm{du}$ CRN trouvée avec le son de blé traduit une légère réduction de l'excrétion urinaire d'azote (Rérat 1978). Enfin la rétention azotée n'est pas modifiée par la nature de lipides, en accord avec les résultats de Reis de Souza (1992).

\section{Activité des enzymes digestives}

Une augmentation du poids du pancréas consécutive à l'incorporation de fibres alimentaires (tableau IV) confirme une stimulation possible de l'activité totale des enzymes comme résultat de l'augmentation de la teneur en fibres du régime (Zebrowska et Low, 1987, Corring et al, 1989). D'autre part, l'adaptation de la lipase à la teneur en lipides du régime est un aspect bien connu chez le porcelet (Mourot et Corring, 1979), en revanche son adaptation à la nature des lipides n'est pas évidente. Ainsi, le remplacement de l'huile d'olive par une graisse saturée n'a pas stimulé l'activité totale et spécifique de la lipase.

\section{Les paramètres biochimiques san- guins}

Nos résultats montrent que la présence de son de blé est sans effet sur la glycémie postprandiale (fig 1) malgré la réduction de la quantité d'amidon ingéré. Ces résultats confirment nos précédentes observations (Bengala Freire et al, 1990) effectuées chez des porcelets de race Large White. Cependant Heppel et Rainbird (1985) ont trouvé chez le porc un effet hypoglycémiant avec des glucides pariétaux aussi différents que la gomme de guar, le son fin de blé et la cellulose purifiée de bois. On peut donc conclure que l'effet hypoglycémiant des glucides pariétaux est fonction de leur nature ou de leurs propriétés chimiques. L'augmentation $(p<0,01)$ de l'urémie consécutive à l'ingestion d'aliment (fig 1) peut traduire une désamination microbienne de l'azote dans le gros intestin en présence d'un excès d'azote des régimes par rapport aux besoins des porcelets de la race Alentejana, lesquels restent encore méconnus. Les résultats relatifs à la variation du taux de cholestérol, des HDL-cholestérol, LDL- 

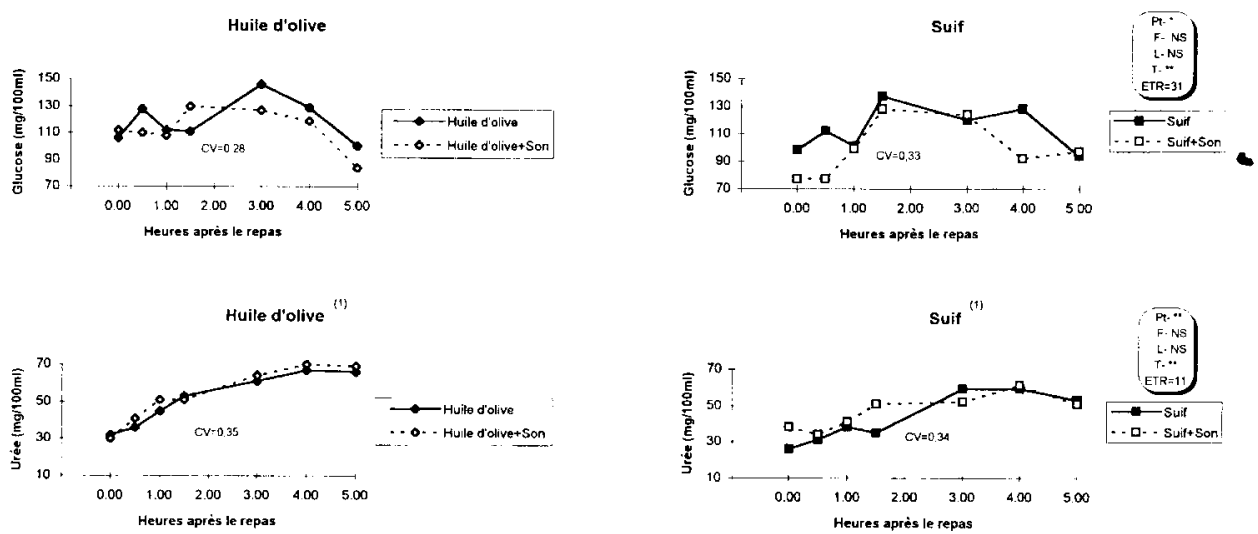

Fig 2. Effet de la nature des lipides et de la présence de fibres dans le régime sur la variation postprandiale du cholestérol et les triglycérides du sang. Effets : Pt : portée, $F$ : taux de fibres, $L:$ nature des lipides, $\mathrm{T}$ : temps après le repas, ETR : écart type de la résiduelle, $\mathrm{CV}$ : coefficient de variation.
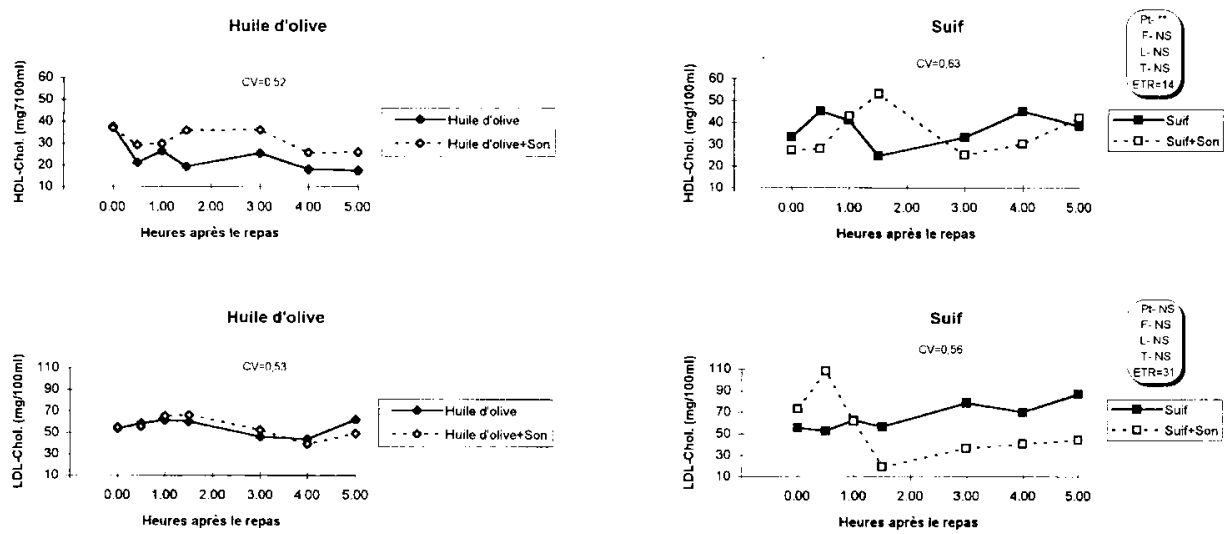

Fig 3. Effet de la nature des lipides et de la présence de fibres dans le régime sur la variation postprandiale du HDL-cholestérol et du LDL-cholestérol du sang. Effets : $\mathrm{Pt}$ : portée, $\mathrm{F}$ : taux de fibres, $\mathrm{L}$ : nature des lipides, $T$ : temps après le repas, ETR : écart type de la résiduelle, CV : coefficient de variation.

cholestérol et des triglycérides doivent être considérés avec prudence en raison de leurs forts coefficients de variation (40 à $60 \%$ ). Cependant nos résultats sur le cholestérol plasmatique exprimés en $\mathrm{mmol} /$ Lsont comparables à ceux rapportés par Beynen et al (1990) sur des animaux Large White. Ainsi, l'inclusion de son de blé ou une ingestion journalière moyenne de $6,5 \mathrm{mg}$ de cholésterol avec le suif n'ont pas modifié le taux de cholestérol plasmatique ni son transport par les lipoprotéines de haute ou basse densité, bien qu'ils soient susceptibles de stimuler la sécrétion biliaire de cholestérol et réduire sa réabsorption intestinale (Corring et al, 1989). 


\section{CONCLUSION}

L'utilisation de lipides riches en acides gras insaturés (huile d'olive) dans le régime augmente significativement la digestibilité fécale apparente des lipides et de l'énergie de la ration de sevrage. La présence d'huile d'olive améliore également la digestibilité fécale apparente de l'azote. De plus, les porcelets de la race Alentejana digèrent dès le sevrage les hémicelluloses du son de blé au niveau de $50-60 \%$. Ces résultats confirment la bonne capacité des jeunes animaux Alentejano pour digérer les régimes riches en fibres et en lipides insaturés, cas des ressources alimentaires des "Montados". Nos données sur la lipémie plasmatique indiquent enfin que les animaux de race Alentejana (génotype gras) ont une bonne capacité endogène pour régulariser le taux de cholestérol sanguin.

\section{REMERCIEMENTS}

Nous remercions le Dr A Resende pour la cathétérisation jugulaire des porcelets et le Dr M João pour la préparation et l'analyse des plasmas. Nous remercions également $M \mathrm{~J}$ António Santos pour la collecte et la préparation des échantillons de fèces et d'urines et Mme L Forte et Mme L Pires pour les analyses pratiquées sur les régimes, les fèces et les urines.

\section{RÉFÉRENCES}

Antunes Correia J C, Oliveira A R (1985) Evolução da consanguinidade em nucleos fechados de porco Alentejano. XXI Jornadas Luso-Espanholas de Genética. Vila Real - Portugal, 24-26 Setembro

Aumaitre A, Rérat A (1968) Digestive enzymes in piglets during weaning: pancreatic lipase. Sess 7, Cont Pap 83 Proc 2nd World Conf Anim Prod, University of Maryland, College Park, MD, États-Unis, 381

Bengala Freire J, Peiniau J, Aumaitre A (1990) Conséquences digestives et métaboliques de l'introduction de glucides pariétaux dans la ration du porcelet sevré. Sciences Alim 10, 293-307
Bergmeyer HU, Gawehn K (1978) Biochemical reagents and samples. Chapter 3. In: Principals of Enzymatical Analysis. 1st edn (HU Bergmeyer, K Gawehn, eds), Verlag Chemieweinhein, New York

Beynen AC, West CE, Spaaij CJK, Huisman J, Van Leeuwen P, Schutte JB, Hackeng WHL (1990) Cholesterol metabolism, digestion rate and postprandial changes in serum of swine fed purified diets containing either casein or soybean protein. J Nutr 120 , 422-430

Braude R, Newport MJ (1973) Artificial rearing of pigs. 4. The replacement of butterfat in a whole milk diet by either beef tallow, coconut oil or soyabean oils. $\mathrm{Br} J$ Nutr 23, 447-455

Campbell RG, Taverner MR (1986) The effects of dietary fiber, source of fat and dietary energy concentration on the voluntary food intake and performance of growing pigs. Anim Prod 43, 327-333

Chabeauti E, Noblet J, Carré B (1991) Digestion of plant cell walls from four different sources in growing pigs. Anim Feed Sci Technol 30, 175-191

Corring T, Juste C, Lhoste EF (1989) Nutritional regulation of pancreatic and biliary secretions. Nutr Res Rev 2, 161-180

Dahlquist A (1964) Method for assay of intestinal disaccharidases. Analyt Biochem 7, 18-25

Drochner W (1984) The influence of changing amounts of crude fibre and pectic components on prececal and postileal digestive processes in the growing pig. $Z$ Tierphysiol Tierernhr Futtermittelkde, Supp $N^{\circ} 14$, $125 \mathrm{p}$

Enders B, Aherne FX, Ozimek L, Spicer H (1988) The effects of fat supplementation on ileal versus fecal fat digestibilities, performance and body composition of weaned pigs. Can J Anim Sci68, 225-231

Eusébio JA, Hays VW, Speer VC, McCall JT (1965) Utilisation of fat by young pigs. J Anim Sci 24, 10011007

Février C, Bourdon D, Aumaitre A (1992) Effects of level of dietary fibre from wheat bran on digestibility of nutrients, digestive enzymes and performance in the European Large White and Chinese Mei Shan pig. $J$ Anim Physiol Anim Nutr 68, 60-72

Frank GR (1982) The utilization of dietary fiber and its effects on the utilization of other dietary components by swine. Graduate College of the University of Illinois, Urbana-Champaign, IL, États-Unis, $120 \mathrm{p}$

Henry $Y$ (1976) Prediction of energy values of feeds for swine from fiber content. Proceedings, First International Symposium Feed Composition, Animal Nutrient Requirements and Computerization of Diets, Logan Utah, Agric Exp Saint, Logan, UT, Ėtats-Unis, 270-281

Heppel LMJ, Rainbird AL (1985) Effect of the physical form of dietary guargum on nutrient absorption in the pig. Proceedings of the 3rd International Seminar on Digestive Physiology in the Pig. Copenhagen, 
16-18 May, Beret Statens Husdrybrugforsog, N $580,58-60$

Inra (1984) L'alimentation des animaux monogastriques: porc, lapin, volailles. Inra, Versailles, $282 \mathrm{p}$

Inra (1987) Répertoire général des aliments, table de composition des corps gras. Inra, Versailles, $167 \mathrm{p}$

Jenkins DJA, Bright-See E, Gibson RS, Josse RG, Kritchevsky D, Peterson RD, Rasper VF (1985) Report of the expert advisory commitee on dietary fibre to the health protection branch health and welfare. Canada, Ministry of National Health and Welfare, Ottawa, Canada, $30 \mathrm{p}$

Jones DB, Hancokk JD, Nelssen JL, Hines RH (1992) Effects of lecithin and lysolecithin on growth performance and nutrient digestibility in weaning pigs. $J$ Anim Sci 70, 3473-3482

Métais $P$, Bieth J (1968) Détermination de l'amylase par une microtechnique. Ann Biol Clin 26, 133-142

Mourot J, Corring T (1979) Adaptation of the lipase-colipase system to dietary lipid content in pig pancreatic tissue. Ann Biol Anim Biochem Biophys 19, 119124

Peiniau J (1984) Les glucides des céréales dans l'alimentation du porcelet sevré : dosage des constituants pariétaux, utilisation digestive et signification nutritionnelle. Thèse ingénieur, Cnam, Paris, $45 \mathrm{p}$

Rathelot J, Julien R, Canini P, Coeroll C, Sarda $L$ (1975) Studies on the effect of the bile salt and colipase on enzymatic lipolysis. Improved method for the determination of pancreatic lipase and colipase. Biochimie $57,1117-1122$

Reboud JP, Ben Abdeljil A, Desnuelle P (1962) Variation de la teneur en enzymes du pancréas du rat en fonction de la composition des régimes. Biochim Biophys Acta 58, 326-337

Reis de Souza TC (1992) Conséquences digestives métaboliques et zootechniques de l'incorporation des lipides dans la ration du porcelet au sevrage. These de doctorat, université de Rennes-I, Rennes, France, $154 \mathrm{p}$

Rérat A (1978) Digestive and absorption of carbohydrates and nitrogenous matters in the hindgut of the omnivorous non ruminant animal. J Anim Sci 46 . 1808-1837

Sas (1989) SAS/STAT User's Guide, Version 6, Fourth Edition, Volume 2, Sas Institut Inc, Cary, NC, $846 \mathrm{p}$

Sethi S, Gibney M J, Williams C M (1993) Postprandial lipoprotein metabolism. Nutr Res Rev 6, 161-183

Van Soest PJ (1963) Use of detergents in the analysis of fibrous feeds. I. Preparation of fiber residue of low nitrogen content. $J$ Ass Off Analyt Chem 46, 825829

Zebrowska T, Low G (1987) The influence of diets based on whole wheat, wheat flour and wheat bran on exocrine pancreatic secretion in pigs. J Nutr 117, 12121216 